\section{OCORRENCIA DE PARACOOPERIA NODULOSA (SCHWARTZ, 1929) EM BUBALUS BUBALIS (LICHTENSTEIN, 1814) DO VALE DO RIBEIRA, ESTADO DE SĀO PAULO}

\author{
MARIA SHIRLEY PIZOLATO OBA \\ Professora Assistente Doutora \\ Instituto de Ciências Biomédicas da USP \\ ARLETE DELL'PORTO \\ Professora Assistente \\ Instituto de Ciências Biomédicas da USP \\ TAKAKO UEKI FUJII \\ Médica Veterinária \\ Delegacia Agrícola de Registro
}

OBA, M.S.P.; DELL'PORTO, A.; FUJII, T.U. Ocorrência de Paracooperia nodulosa (Schwartz, 1929) em Bubalus bubalis (Lichtenstein, 1814) do Vale do Ribeira, Estado de São Paulo. Rev. Fac.Med.vet.Zootec.Univ.S. Paulo, 18(2): 97-103, 1981

RESUMO: Sintetizou-se o histórico da introdução do búfalo no Brasil e registrou-se o encontro de Paracooperia nodulosa em bubalinos do Vale do Ribeira, Estado de São Paulo, descrevendo as lesð̋es macroscópicas encontradas no intestino delgado bem como a morfologia de machos e fêmeas adultos do parasita.

UNITERMOS: Paracooperia nodulosa*; Bubal us bubalis*; Trichostrongylidae*.

\section{INTRODUÇÃO}

\section{O BÚFALO}

A chegada do búfalo no Brasil data do início deste século, sendo portanto bastante recente. Porém, os dados são incertos; para alguns criadores marajoaras esse gado vem sendo criado na ilha de Marajó desde 1890, outros porém calculam que sua entrada tenha ocorrido por volta de 1902 e 1903.

No sul do país, as primeiras entradas datam de 1908 na região meridional, quando a Casa Herm. Stoltz \& Cia. inaugurou em 12 de novembro de 1907 no antigo Jardim Zoológico do Rio de Janeiro, uma estação zootécnica, destinada a abrigar e exibir reprodutores bovinos, animais silvestres e espécies consideradas exóticas, as quais sejam zebras e búfalos indianos. Posteriormente, em 1919, um criador mineiro em visita à Índia, trouxe 2 casais de búfalos para o Brasil. Em 1920, outros 2 casais aqui chegaram, também no Estado de Minas Gerais. $\hat{\mathrm{E}}$ desses 4 casais que descende grande parte do rebanho bubalino dos Estados de Minas Gerais e São Paulo.

O introdutor da espécie em São Paulo, foi o Cel. Antonio Jacinto Sobrinho, que levou para Franca alguns dos primeiros produtos nascidos em Cássia, filhos de animais importados. O rebanho cresceu e atingiu a cidade de Vale do Sapucai, tornando-se esta o primeiro centro de criação de bubalinos no Estado de São Paulo. A dispersão por áreas outras do Estado, teve início por volta de 1950.

Em torno de 1920, também chegaram a São Paulo, bubalinos de origem italiana, tendo sido instalados no então município de Santa Rosa, na regiđo Mogiana. Posteriormente, em 1948, mais 22 animais chegam ao sul do Estado, em São Miguel Arcanjo. Produtos dessas propriedades originaram dois núcleos de criação: Ribeirão Claro (norte do Paraná) e São José dos Campos (Vale do Paraiba), sendo este último misto.

Também em outros estados, tais como Mato Grosso do Sul, Mato Grosso do Norte, Paraná, Santa Catarina e Rio de Janeiro, são encontrados núcleos de criação de bubalinos.

\section{O PARASITO}

Em 1928, Schwartz trabalhando nas ilhas Filipinas, ao necropsiar em carabao (Bubalus bubalis), observou pela primeira vez formações nodulares na parede do intestino delgado desse animal e denominou o agente causal como Cooperia nodulosa. Posteriormente, Monnig (1931) no exame post-mortem de uma gazela (Antidorscas marsupialis) descreve uma outra espécie como Cooperia serrata, na África do Sul. Justificava ele a nova denominação em função da diferença do número de formações semilunares presentes nos espículos de ambas as espécies. Em 1933, DAUBNEY 5 1933, descreve como C. serrata um parasito de carneiro da 
Colonia do Kenya. TRAVASSOS ${ }^{11} 1935$, revisando o gênero Cooperia, cria um novo gênero Paracooperia, considerando Paracooperia serrata (sin. Cooperia serrata Monnig, 1931) como espécie tipo; considerou ainda o espécime descrito por DAUBNEY ${ }^{5}$ 1933, distinto de P. nodulosa e P. serrata denominando-a P. daubneyi. LEROUX ${ }^{7}$ 1936, desconhecendo o trabalho de TRAVASSOS11 1935, propøe um novo gênero Schwartziella para aqueles espécimes descritos por Schwartz e Monnig, respectivamente. Em 1950, LEROUX $^{7}$ 1950, registra uma nova espécie P. mazabukae encontrada no duodeno de um ruminante silvestre, o oribi (Ourebia ourebi) da região de Mazabuka no norte da Rodesia. Ainda neste trabalho, ele propõe que o parasita descrito em 1938 por Matoff como Schwartziella nodulosa encontrada no intestino delgado e ceco dos búfalos da Bulgária, seja denominada P. matoffi.

\section{MATERIAL E METODOS}

Os animais necropsiados pertenciam aos planteis de bubalinos do Vale do Ribeira.

Por dissecção dos nódulos existentes nas paredes do duodeno, jejuno e íleo de bubalinos obtivemos os exemplares adultos - jovens. Do conteúdo do lumen intestinal foram obtidos os adultos machos e fêmeas.

Estes helmintos foram conservados em solução de formol acético. Destes, 60 foram utilizados para tomadas de medidas. Na verificação da morfologia utilizamos lactofenol como clarificante. Os desenhos foram obtidos em câmara clara.

\section{ASPECTOS MACROSCÓPICOS DAS LESÕES}

Nódulos do tamanho de semente de ervilha distribuiam-se ao longo do intestino delgado, com concentração maior no jejuno seguida pelo ileo e duodeno. Nas porções intestinais intensamente parasitadas um exsudato muco-fibrinoso estava presente. Estes nódulos apresentavam coloração amarelada e eram facilmente visíveis tanto na camada mucosa quanto na camada serosa. Alguns deles apresentavam um foco hemorrágico na parede central. De cada nódulo extraiu-se um ou dois exemplares, na fase adulta do desenvolvimento.

\section{SINTOMATOLOGIA}

Os animais portadores dessa parasitose apresentavam um histórico clínico de diarreia constante, anemia progressiva e emaciação.

\section{DIAGNÓSTICO}

Os parasitos obtidos à partir dos nódulos eram ainda jovens, porém perfeitamente diferenciáveis sexualmente.
Aqueles que foram colhidos do lumen, estavam completamente maduros pois as fêmeas apresentavam alguns ovos em desenvolvimento e outros perfeitamente formados nas alças uterinas.

Morfologicamente o gênero Paracooperia descrito por TRAVASSOS 12 1935, apresenta na extremidade cefálica uma expansశ̃o cuticular bem desenvolvida, com ligeira constricção próxima à extremidade anterior. Esta expansão cuticular apresenta ainda estriaçðes transversais as quais começam logo abaixo da abertura bucal. A cutícula ao longo do corpo, por sua vez, também apresenta estriaçoes transversais em número de 10 estrias que começam logo abaixo da vesícula cefálica. $O$ esôfago é do tipo claviforme e apresenta $o$ anel nervoso na sua porção medial. $O$ poro excretor abre-se na altura do anel nervoso. $O$ intestino por outro lado é reto e simples.

MACHOS - os machos, jovens e adultos, apresentam bolsa copuladora bem desenvolvida, com 2 espículos caracterizados pela presença de formações semilunares em número de 7 a 10 dirigidos para a linha mediana e tendo na extremidade distal uma formação ligeiramente globular donde parte uma estrutura em forma de espinho (Fig. 1). A bolsa copuladora é sustentada por raios com a seguinte disposição: raio lateral anterior mais delgado que o ventro-lateral (mais grosso de todos); seguindo-se em grossura o lateral anterior, lateral médio e lateral posterior (ambos com a mesma espessura); dorsal externo grosso na porção proximal e afilado distalmente. Os raios pares terminam próximo à margem bursal exceto o dorsal externo. $O$ raio dorsal é longo e fino, apresenta-se furcado e cada ramo secundário tem 2 ramos obtusos e um ramo lateral mais ou menos normal ao eixo do nematóide. Neste gênero observa-se ainda ausência do gubernáculo (TRAVASSOS12 1937 e CHAUHAN e PANDE 3 1972) Fig. 2.

FÊMEAS - tanto as fêmeas jovens quanto as adultas apresentam um "flap" linguiforme bem desenvolvido no terço médio do corpo (Fig. 3), anterior à abertura vulvar, levemente curvado lateralmente e suportado por uma estru. tura central interna semelhante a raios de guarda-chuva. A vagina é curta e apresenta um ovejector comum com esfincteres musculares em ambas as extremidades os quais se continuam por 2 alças uterinas que terminam em ovários. A cauda é cônica, com extremidade ligeiramente arredondada e apresenta estrias transversais (Fig. 4).

Destes adultos foram realizadas medidas em 60 exemplares machos e 60 fêmeas, estando os valores médios obtidos sumariados no QUADRO I, juntamente com os dados de CHAUHAN e PANDE 3 1972; VENKATARATNAM 13 1964 e TRAVASSOS12 1937.

\section{DISCUSSÃO}

CHAUHAN e PANDE 3 1972, verificaram que esta parasitose apresenta fenômeno de incidência sasonal assim dis- 
tribuida: a porcentagem dos nódulos parasitados é máxima no verão e mínima no inverno, enquanto que os vermes adultos livres no lúmen intestinal são encontrados em maior quantidade no inverno e menor no verão. Os dados por nós obtidos não podem ser analisados e comparados com os daqueles autores visto termos trabalhado com material de apenas duas necrópsias ocorridas em épocas diferentes (fevereiro e setembro), porém o que se pode observar é que no material da necrópsia realizada no mês de fevereiro havia maior quantidade de vermes adultos livres no lúmen do que a realizada no mês de setembro.

\section{CONCLUSÃO}

Dada a localização e a morfologia dos parasitos, com base nos trabalhos de PATNAIK e PANDE 8 1963; VENKATARATNAM 13 1964; SUNDARAM e cols. 10 1968; ACHUTAN 1970 e CHAUHAN e PANDE 2,3 1972, concluimos tratar-se de Paracooperia nodulosa.

OBA, M.S.P.; DELL'PORTO, A.; FUJII, T.U. Occurrence of Paracooperia nodulosa (Schwarts, 1929) in Bubal us bubalis (Lichtenstein, 1814) in the Ribeira Valley, State of São Paulo, Brazil. Rev.Fac.Med.vet.Zootec.Univ.S. Paulo, 18(2): 97-103, 1981.

SUMMARY: It was summarised the history about the water buffalo introduction in Brazil, and registered the Paracooperia nodulosa in buffalo of the Ribeira Valley, State of São Paulo. It was described the macroscopical lesions in the wall of the small intestine as well as the morphology of the male and female adult parasites.

UNITERMS: Paracooperia nodulosa * ; Bubal us bubalis"; Trichostrongylidae*.

\section{REFERENCIAS BIBLIOGRÁFICAS}

1- ACHUTAN, H.N. Paracooperiosis in buffalo-calves due to Paracooperia matoffi (Matoff, 1938) Le Roux, 1950. Indian vet.J., 42:451-3, 1970.

2- CHAUHAN, P.P.S. \& PANDE, B.P. Studies on paracooperial infection in buffalo calves. I. Seasonal incidence and development of pre-parasitic stages with a new host record in a black buck. Indian J.anim.Sci., 42(12):1033-8, 1972.

3- ChAUHAN, P.P.S. \& PANDE, B.P. Studies on paracooperid infection in buffalo calves. II. Mor- phology of parasitic juveniles and du uils will remarks. on validity of Paracooperia matoffi. Indian J.anim.Sci., 42(11):919-29, 1972.

4- CHAUHAN, P.P.S. \& PANDE, B.P. Studies on paracooperid infection in buffalo calves. III. Nodules and their histopathology. Indian J.anim. Sci., 42(11):930-4, 1972.

5- DAUBNEY, R. Trichostrongylid nematodes from sheep in Kenya. Parasitology, 25:224-41, 1933.

6- LeROUX, P.L. On Schwartziella, a new nematode Genus for Cooperia nodulosa Schwartz, 1928. J.Helminth., 14:113-8, 1936.

7- LeROUX, P.L. A trichostrongylid, Paracooperia mazabukae sp. nov., from a wild ruminant, the Oribi, in Northern Rhodesia. J.Helminth., 24: 28-32, 1950 .

8- PATNAIK, M.M. \& PANDE, B.P. Notes on the helmintic infestations encountered in one month old buffalo calves. Indian vet.J., 40: 128-33, 1963.

9- STARKE, W.A.; MACHADO, R.Z.; ZOCOLLER, M.C. Infestação natural por nematóides parasitas de búfalos nascidos no município de Andradina - São Paulo. CONGRESSO BRASILEIRO DE PARASITOLOGIA, 5., Rio de Janeiro, 1980. Resumos.

10- SUNDARAM, R.K.; SILVASUBRAMANIYAM, M. S.; IYER, R.P.; PETER, C.T. Incidence of Paracoopería matoffi (Matoff, 1938), LeRoux, 1950 in Indian buffaloes. Indian vet.J., 45:2231,1968 .

11- TRAVASSOS, L. Alguns novos gêneros e espécies de Trichostrongylidae. Rev.Med. Cirurgia Brasil., 43(11):345-61, 1935.

12- TRAVASSOS, L. Revisão da família Trichostrongylidae Leiper, 1912. Monographias do Inst. Oswaldo Cruz, 1:49, 1937.

13- VENKATARATNAM, A. Incidences of paracooperiasis in Andhra Pradesh. Indian vet.J., 41:349. $51,1964$.

Recebido para publicação em: 14-04-81 Aprovado para publicação em: 04-06-81 
QUADRO I - Tamanho médio em milímetros das diferentes estruturas de machos e fêmeas de $\mathbf{P}$. nodulosa, segundo diversos autores.

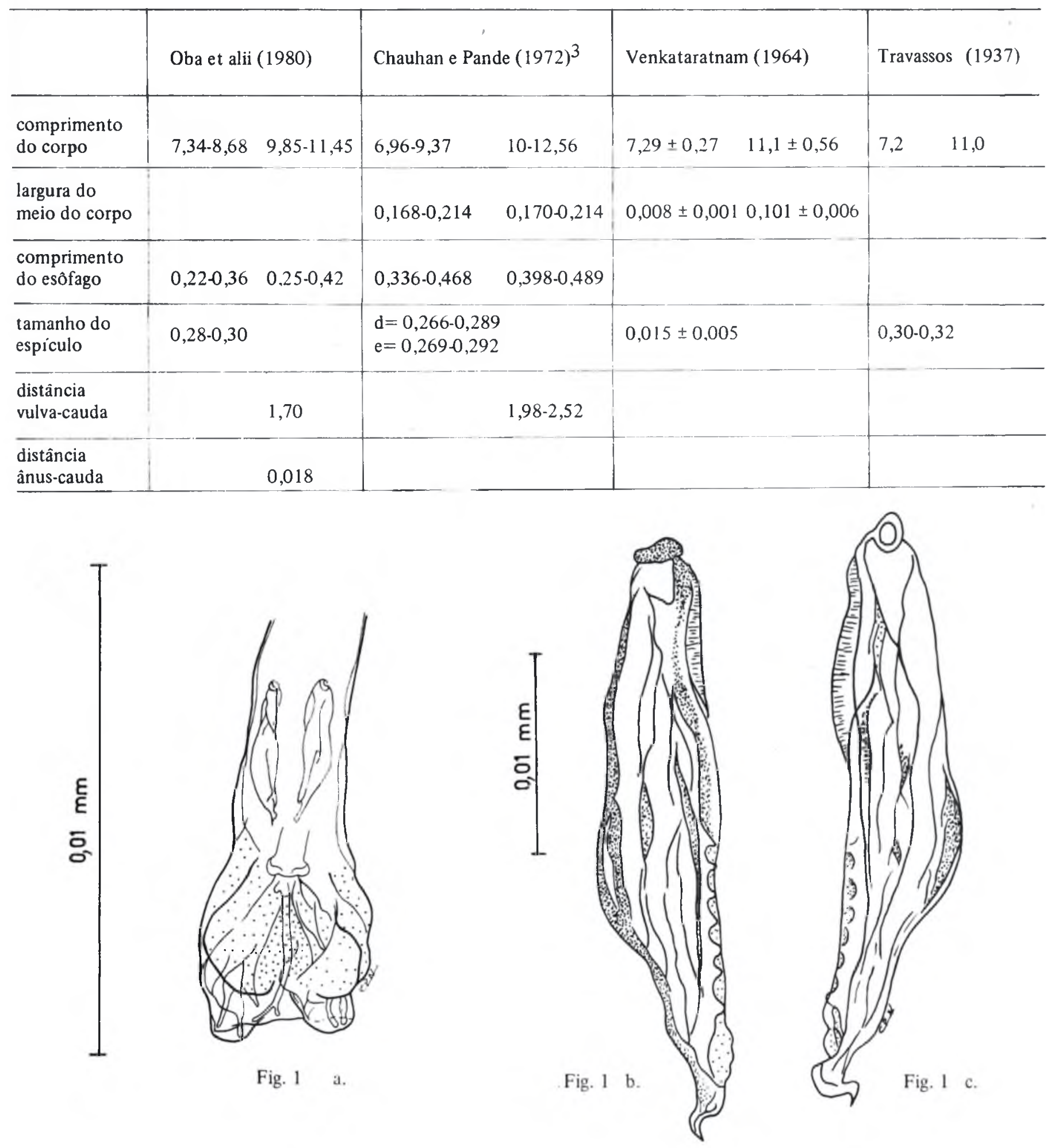

Fig. 1 - Machos adultos de Paracooperia nodulosa. a. Bolsa copuladora. b. c. Espículos. 


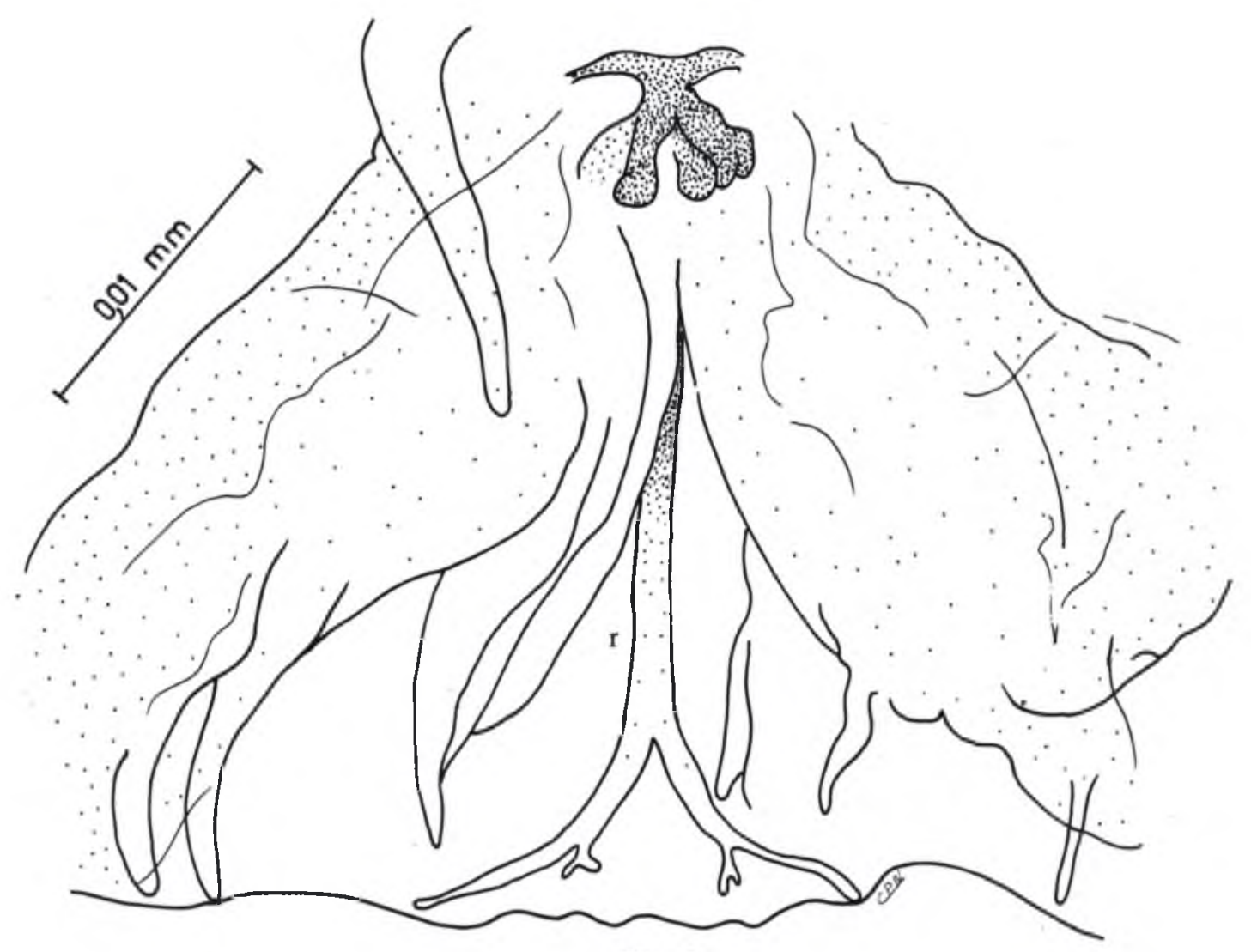

Fig. 2

Fig. 2 - Detalhes da bolsa copuladora . r. raio dorsal. 


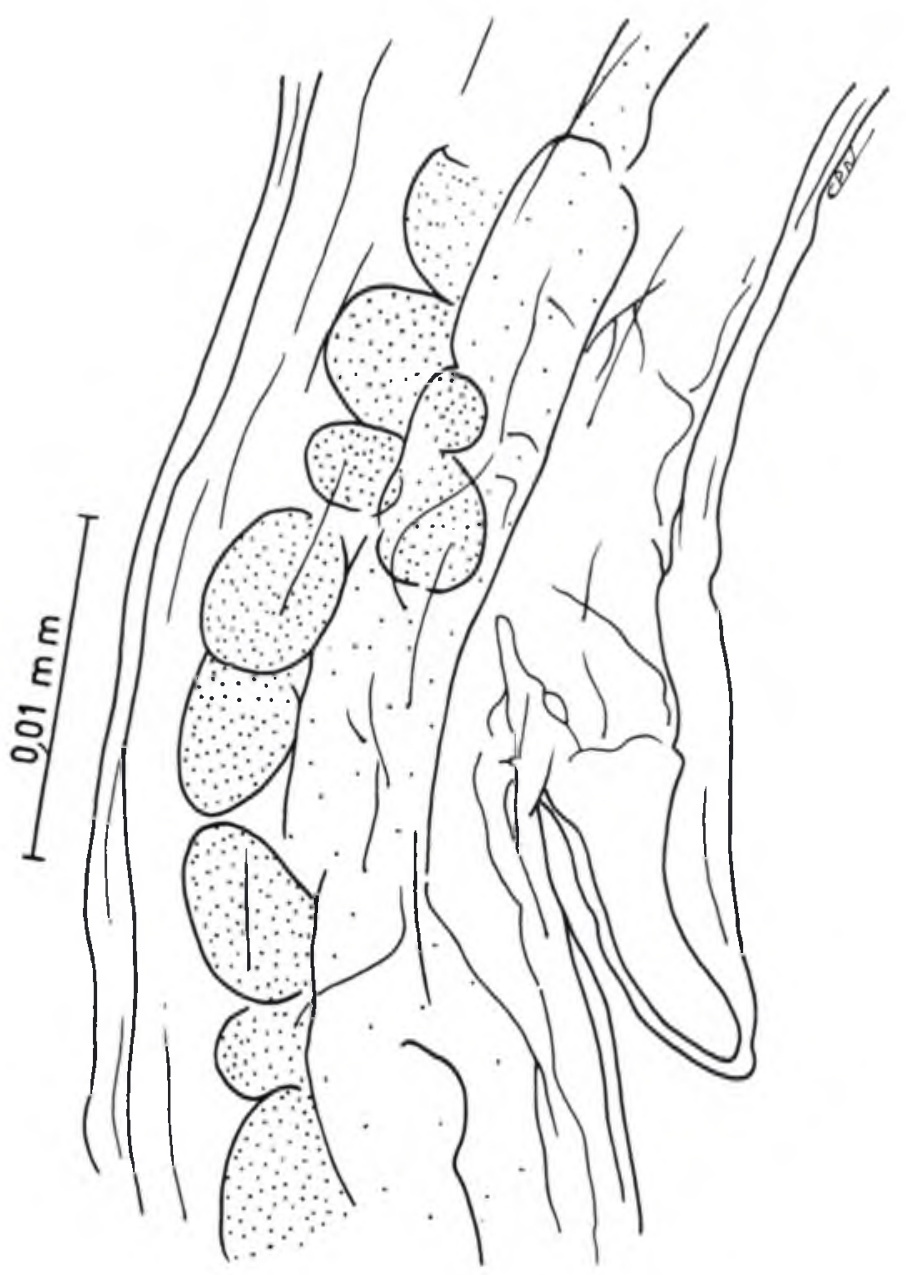

Fig. 3 - Região vulvar, flap de fêmeas adultas. 


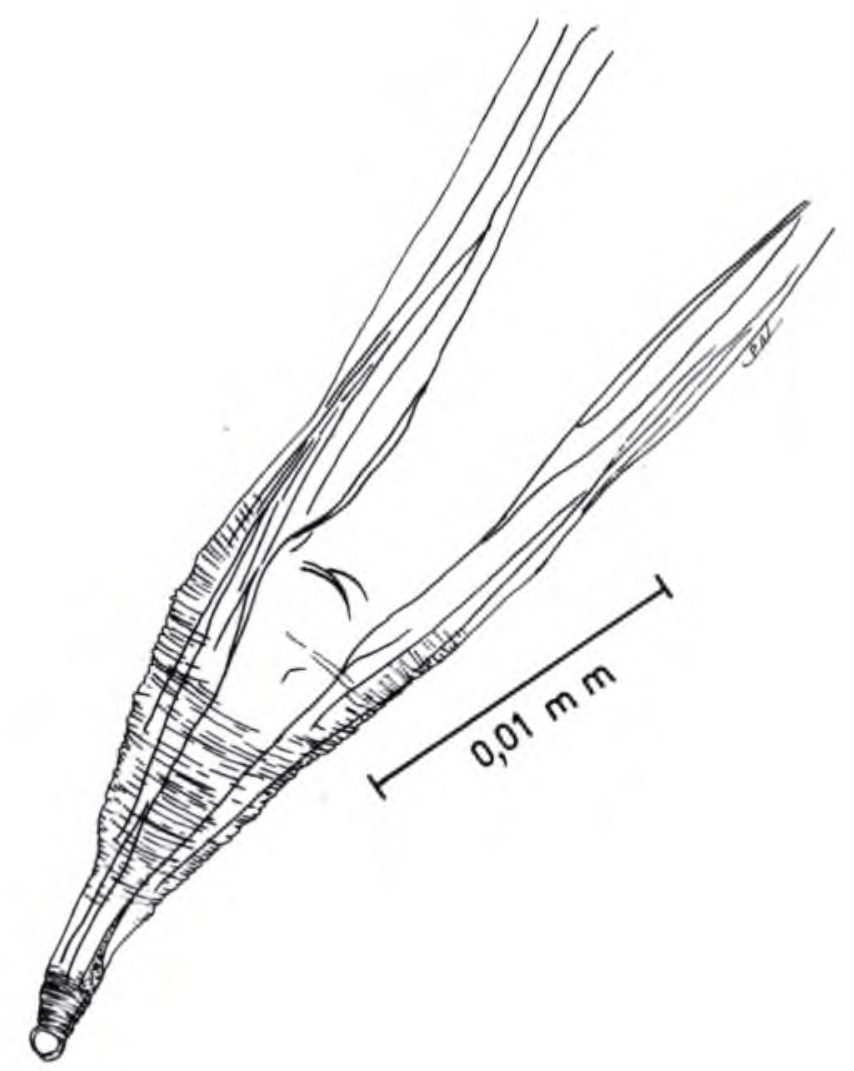

Fig. 4 - Paracooperia nodulosa - extremidade posterior da fêmea adulta. 